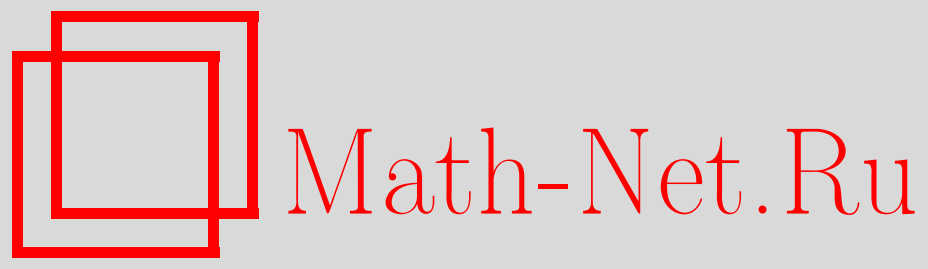

Е. Ю. Смирнов, Разрешения особенностей для многообразий Шуберта в двойных грассманианах, Функи. анализ и его прил., 2008, том 42, выпуск $2,56-67$

DOI: https://doi.org/10.4213/faa2902

Использование Общероссийского математического портала MathNet.Ru подразумевает, что вы прочитали и согласны с пользовательским соглашением

http://www. mathnet.ru/rus/agreement

Параметры загрузки:

IP : 52.90 .164 .192

26 апреля 2023 г., 16:17:06

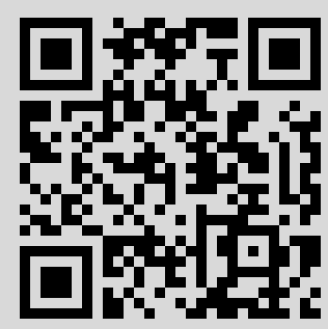


Функиионалъный анализ и его приложения

2008, т. 42, вып. 2, с. 56-67

УДК $512.743+512.761 .2$

\title{
Разрешения особенностей для многообразий Шуберта в двойных грассманианах
}

\author{
(c) 2008. Е. Ю. Смирнов
}

\section{§1. Введение}

Пусть $V$ - конечномерное векторное пространство над произвольным полем $\mathbb{K}$.

Мы будем описывать пары подпространств пространства $V$ фиксированных размерностей $k$ и $l$ с точностью до действия группы невырожденных верхнетреугольных матриц $B \subset \mathrm{GL}(V)$. Другими словами, мы описываем $B$-орбиты на прямом произведении $X=\operatorname{Gr}(k, V) \times \operatorname{Gr}(l, V)$ двух грассмановых многообразий. Разложение многообразия $X$ на $B$-орбиты является аналогом шубертовского разложения для грассманианов и разложения Эресмана-Брюа для многообразий полных флагов.

Комбинаторное описание $B$-орбит на $X$ приводится (как частный случай некоторой более общей задачи) в работе Мадьяра, Веймана и Зелевинского [7] и существенно использует теорию колчанов. Приводимое ниже описание опирается лишь на элементарную линейную алгебру и не использует результатов из [7]. Оно является обобщением описания орбит в симметрическом пространстве $\mathrm{GL}_{k+l} /\left(\mathrm{GL}_{k} \times \mathrm{GL}_{l}\right)$, приведенного в диссертации Пина [8].

Нас также интересуют замыкания $B$-орбит в $X$. Они являются аналогами многообразий Шуберта в грассманианах. Особенности многообразий Шуберта хорошо изучены: для них имеются разрешения, построенные Боттом и Самельсоном; известно, что многообразия Шуберта являются нормальными, а их особенности рациональны; множества их особых точек могут быть явно описаны. Подробнее об этом можно прочитать, например, в лекциях [2] и книге [6]. Таким образом, было бы естественно задать те же вопросы (разрешение особенностей, нормальность, рациональность) и для замыканий $B$-орбит в $X$. В настоящей работе строятся разрешения особенностей для этих многообразий.

Мотивировкой для рассмотрения этих вопросов является также недавняя статья Бобинского и Звары [3], в которой доказывается, что особенности замыканий орбит в представлениях колчанов типа $D$ эквивалентны особенностям многообразий Шуберта в двойных грассманианах.

Автор благодарит Мишеля Бриона за постоянное внимание к работе, а также Э. Б. Винберга и Д. А. Тимашёва за ценные обсуждения и замечания.

\section{§2. Описание орбит}

2.1. Обозначения. Пусть $V$ - векторное пространство размерности $n$ над полем $\mathbb{K}, k, l<n$ - натуральные числа. Результаты, приведенные в этом параграфе, верны над произвольным основным полем; в $\S 3,4$ требуется, чтобы поле $\mathbb{K}$ было алгебраически замкнутым. Прямое произведение $\operatorname{Gr}(k, V) \times \operatorname{Gr}(l, V)$ 
будет обозначаться через $X$. Как правило, мы не будем различать точки в $X$ и соответствующие конфигурации подпространств $(U, W)$, где $U, W \subset V$, $\operatorname{dim} U=k, \operatorname{dim} W=l$.

Мы фиксируем борелевскую подгруппу $B$ в $\mathrm{GL}(V)$. Через $V_{\bullet}=\left(V_{1}, \ldots, V_{n}=\right.$ $V$ ) будет обозначаться полный флаг в $V$, неподвижный относительно группы $B$.

Комбинаторное описание. В этой части вводятся комбинаторные объекты, параметризующие пары подпространств с точностью до действия группы $B$. А именно, пары подпространств параметризуются тройками, состоящими из двух диаграмм Юнга, которые содержатся в прямоугольниках размера $k \times(n-k)$ и $l \times(n-l)$ соответственно, и инволютивной перестановки из $S_{n}$.

Параллельно с введением этих комбинаторных объектов мы строим некоторые «канонические» базисы пространств $U, W$ и $V$.

Предложение 1. (i) Существуют такие упорядоченные базисы $\left(u_{1}, \ldots, u_{k}\right)$, $\left(w_{1}, \ldots, w_{l}\right)$ u $\left(v_{1}, \ldots, v_{n}\right)$ пространств $U, W u V$ соответственно, что

- $V_{i}=\left\langle v_{1}, \ldots, v_{i}\right\rangle$ для всех $i \in\{1, \ldots, n\}$ (угловые скобки обозначают линейную оболочку векторов);

- $u_{i}=v_{\alpha_{i}}$, гдe $i \in\{1, \ldots, k\} u\left\{\alpha_{1}, \ldots, \alpha_{k}\right\} \subset\{1, \ldots, n\}$;

- все $w_{i}$ суть либо базисные векторы пространства $V$, либо векторы с двухэлементным «носителем»: $w_{i}=v_{\beta_{i}}$ или $w_{i}=v_{\gamma_{i}}+v_{\delta_{i}}$, где $\gamma_{i}>\delta_{i}$; кроме того, в последнем случае $v_{\gamma_{i}} \in U\left(\right.$ m. e. $\left.\left\{\gamma_{1}, \ldots, \gamma_{r}\right\} \subset\left\{\alpha_{1}, \ldots, \alpha_{k}\right\}\right)$.

- все числа $\beta_{i}$ (соответственно $\gamma_{i}, \delta_{i}$ ) различны межсду собой; кроме того, все $\delta_{i}$ отличны от всех $\alpha_{i}$.

(ii) $B$ обозначениях $n$. (i) рассмотрим перестановку $\sigma \in S_{n}$, получаемую как произведение транспозищий $\left(\delta_{i}, \gamma_{i}\right)$. Поскольку носители транспозищий не пересекаются, результат их перемножения не зависит от порядка сомножителей.

Тогда для данной пары подпространств $(U, W)$ множества $\bar{\alpha}=\left\{\alpha_{1}, \ldots, \alpha_{k}\right\}$, $\bar{\beta}=\left\{\beta_{1}, \ldots, \beta_{l-r}\right\}, \bar{\gamma}=\left\{\gamma_{1}, \ldots, \gamma_{r}\right\}$ и перестановка $\sigma$ не зависят от выбора базисов в $U, W u V$.

Доказательство. (i) Доказательство проводится индукцией по $n$.

При $n=1$ утверждение очевидно.

Индуктивный переход от $n-1 \mathrm{k} n$. Возьмем ненулевой вектор $v_{1} \in V_{1}$ и рассмотрим следующие случаи:

- $v_{1} \notin U+W$. Пусть $\bar{V}=V /\left\langle v_{1}\right\rangle$. Возьмем в этом факторпространстве флаг $\bar{V}_{\bullet}=\left(\bar{V}_{2} \subset \cdots \subset \bar{V}_{n}\right)$ и рассмотрим образ $(\bar{U}, \bar{W})$ конфигурации $(U, W)$ при факторизации. Оба полученных подпространства будут изоморфны исходным: $\bar{U} \cong U$ и $\bar{W} \cong W$. Теперь применим предположение индукции к данной конфигурации следующим образом. Выберем в $\bar{U}, \bar{W}$ и $\bar{V}$ упорядоченные базисы $\left\{\bar{u}_{1}, \ldots, \bar{u}_{k}\right\},\left\{\bar{w}_{1}, \ldots, \bar{w}_{l}\right\}$ и $\left\{\bar{v}_{1}, \ldots, \bar{v}_{n-1}\right\}$, после чего выделим $\bar{V}$ в $V$ прямым слагаемым: $\imath: \bar{V} \hookrightarrow V$. «Поднимем» указанные базисные векторы из $\bar{V}$ в $V$ следующим образом: $u_{i}=\imath\left(\bar{u}_{i}\right), w_{i}=\imath\left(\bar{w}_{i}\right), v_{i}=\imath\left(\bar{v}_{i-1}\right)$. Получим искомую тройку базисов.

- $v_{1} \in U, v_{1} \notin W$. Положим $u_{1}=v_{1}$ и вновь применим предположение индукции к факторпространству $\bar{V}=V /\left\langle v_{1}\right\rangle$ с флагом $\bar{V}_{\bullet}$ и конфигурации подпространств $(\bar{U}, \bar{W})$, получающихся из $U$ и $W$ при факторизации. В этом случае $\operatorname{dim} \bar{U}=\operatorname{dim} U-1$. После этого опять поднимем полученные векторы из $\bar{U}, \bar{W}$ и $\bar{V}$ в $V$. 
- Случай $v_{1} \notin U, v_{1} \in W$ разбирается аналогично предыдущему (полагаем $\left.w_{1}=v_{1}\right)$.

- Если $v_{1} \in U \cap W$, положим $u_{1}=w_{1}=v_{1}$ и вновь применим предположение индукции.

- Наиболее интересен последний случай: $v_{1} \in U+W$, но $v_{1} \notin U$ и $v_{1} \notin W$. Тогда рассмотрим множество векторов $S=\left\{v \mid v \in U, v_{1}+v \in W\right\}$. Поскольку $v_{1}$ принадлежит $U+W$, это множество непусто. Пусть теперь $j$ - минимальное число, для которого $V_{j}$ содержит векторы из $S$, и $v_{j} \in V_{j} \cap S$. Положим $u_{1}=v_{j}$, $w_{1}=v_{1}+v_{j}$. Теперь применим предположение индукции к $(n-2)$-мерному пространству $\bar{V}=V /\left\langle v_{1}, v_{j}\right\rangle$, паре подпространств $\bar{U}=U /\left\langle v_{1}, v_{j}\right\rangle, \bar{W}=W /\left\langle v_{1}, v_{j}\right\rangle$ и флагу

$$
\bar{V}_{\bullet}=V_{2} / V_{1} \subset \cdots \subset V_{j-1} / V_{1}=V_{j} /\left\langle v_{1}, v_{j}\right\rangle \subset V_{j+1} /\left\langle v_{1}, v_{j}\right\rangle \subset \cdots \subset V_{n} /\left\langle v_{1}, v_{j}\right\rangle .
$$

«Подъем» базисных векторов из $\bar{V}$ в $V$ осуществляется так:

$$
v_{i}=\imath\left(\bar{v}_{i-1}\right) \quad \text { при } i \in[2, j-1], \quad v_{i}=\imath\left(\bar{v}_{i-2}\right) \quad \text { при } i \in[j+1, n],
$$

где $\imath$, как и прежде, обозначает некоторое вложение $\bar{V}$ в $V$. Векторы $v_{1}$ и $v_{j}$ уже были определены ранее.

(ii) Предположим, что для конфигурации $(U, W)$ существуют две тройки упорядоченных базисов $\left(\left(u_{1}, \ldots, u_{k}\right),\left(w_{1}, \ldots, w_{l}\right),\left(v_{1}, \ldots, v_{n}\right)\right)$ и $\left(\left(u_{1}^{\prime}, \ldots, u_{k}^{\prime}\right)\right.$, $\left.\left(w_{1}^{\prime}, \ldots, w_{l}^{\prime}\right),\left(v_{1}^{\prime}, \ldots, v_{n}^{\prime}\right)\right)$, которые удовлетворяют условиям п. (i) и для которых либо соответствующие этим тройкам базисов наборы $(\bar{\alpha}, \bar{\beta}, \bar{\gamma})$ и $\left(\bar{\alpha}^{\prime}, \bar{\beta}^{\prime}, \bar{\gamma}^{\prime}\right)$, либо перестановки $\sigma$ и $\sigma^{\prime}$ отличны друг от друга.

Множество $\bar{\alpha}$ может быть описано следующим образом: $i \in \bar{\alpha}$ тогда и только тогда, когда $\operatorname{dim} U \cap V_{i}>\operatorname{dim} U \cap V_{i-1}$. Следовательно, $\bar{\alpha}=\bar{\alpha}^{\prime}$.

Аналогично доказывается, что $\bar{\beta} \cup \bar{\gamma}=\bar{\beta}^{\prime} \cup \bar{\gamma}^{\prime}$.

Теперь докажем, что $\sigma=\sigma^{\prime}$. Тем самым доказательство будет закончено, поскольку $\bar{\beta}=\{j \in \bar{\beta} \cup \bar{\gamma} \mid \sigma(j)=j\}$.

Пусть $j$ - минимальное число из $\beta \cup \gamma$, для которого $\sigma(j) \neq \sigma^{\prime}(j)$. Предположим, что $\sigma(j)<\sigma^{\prime}(j)$. Возможны два варианта:

(a) $i:=\sigma^{\prime}(j) \neq j$. Заметим, что $i \notin \bar{\alpha}$. Рассмотрим подпространство

$$
\begin{aligned}
\widetilde{V}=\left(U \cap V_{j}\right)+V_{i-1} & =\left\langle v_{s}, v_{\alpha_{i}} \mid s \leqslant i-1, \alpha_{i} \in \bar{\alpha} \cup[i, j]\right\rangle \\
& =\left\langle v_{s}^{\prime}, v_{\alpha_{i}}^{\prime} \mid s \leqslant i-1, \alpha_{i} \in \bar{\alpha}^{\prime} \cup[i, j]\right\rangle .
\end{aligned}
$$

Пусть $R=\{r \in \bar{\beta} \cup \bar{\gamma} \mid r, \sigma(r) \in[1, i-1] \cup(\bar{\alpha} \cap[i, j])\}$ и $R^{\prime}=\{r \in \bar{\beta} \cup \bar{\gamma} \mid$ $\left.r, \sigma^{\prime}(r) \in[1, i-1] \cup(\bar{\alpha} \cap[i, j])\right\}$. Легко убедиться, что

$$
\operatorname{dim} \widetilde{V} \cap W=\# R=\# R^{\prime} .
$$

Но $\sigma(r)=\sigma^{\prime}(r)$ для всех $r \in[1, j-1]$, а $j$ принадлежит множеству $R$ и не принадлежит $R^{\prime}$. Значит, количество элементов в этих двух множествах различно, что и дает искомое противоречие.

(b) Если $\sigma^{\prime}(j)=j$, положим $i=\sigma(j)$ и будем действовать аналогично тому, как действовали в п. (а).

Теперь введем комбинаторную конструкцию, параметризующую конфигурации подпространств. А именно, по конфигурации мы построим пару диаграмм Юнга, некоторые клетки в которых будут отмечены. 
Рассмотрим пару подпространств $(U, W)$. Построим по этой паре, как описано в предложении 1 , множества $\bar{\alpha}, \bar{\beta}, \bar{\gamma}$ и инволюцию $\sigma$. Рассмотрим прямоугольник размера $k \times(n-k)$ и построим путь из его левого нижнего угла в правый верхний следующим образом: $j$-е звено пути будет вертикальным, если $j$ принадлежит $\bar{\alpha}$ (т. е. если $v_{j}$ равняется какому-то из $u_{i}$ ), и горизонтальным в противном случае. Этот путь ограничивает снизу первую диаграмму Юнга.

Вторая диаграмма будет содержаться в прямоугольнике размера $l \times(n-l)$. Мы снова построим ограничивающий ее путь: $j$-е звено пути будет вертикальным, если $j \in \bar{\beta} \cup \bar{\gamma}$, и горизонтальным в противном случае.

Если $j \in \bar{\gamma}$, то звено пути с номером $\sigma(j)$ горизонтально. Из этого также следует, что $j$-е звено пути, ограничивающего первую диаграмму, вертикально, а $\sigma(j)$-е горизонтально. Возьмем в каждой из диаграмм клетку, находящуюся над $\sigma(j)$-м звеном и слева от $j$-го звена, и поместим в этих клетках по точке.

Назовем такую пару диаграмм отмеченной парой.

Пример. Пусть $n=9, k=4, l=3$. Предположим, что $\bar{\alpha}=\{3,5,6,9\}, \bar{\beta}=$ $\{2,5\}, \bar{\gamma}=\{9\}, \sigma=(7,9)$. Тогда соответствующая отмеченная пара диаграмм выглядит так:
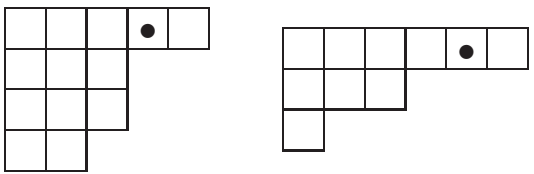

Замечание. Отметим, что построенные диаграммы (как диаграммы без точек) совпадают с диаграммами, которые соответствуют клеткам Шуберта, содержащим точки $U \in \operatorname{Gr}(k, V)$ и $W \in \operatorname{Gr}(l, V)$. (Соответствие между диаграммами Юнга и клетками Шуберта в грассманиане описано, например, в книгах [4] или [6]).

2.3. Стабилизаторы и размерности орбит. Найдем стабилизатор $B_{(U, W)}$ данной конфигурации подпространств $(U, W)$.

Предложение 2. В обозначениях предложения 1 стабилизатор конфигуращии $(U, W)$, записанный в базисе $\left(v_{1}, \ldots, v_{n}\right)$, состоит из верхнетреугольных матрич, $A=\left(a_{i j}\right) \in B$, удовлетворяюших следующим условиям:

(a) $a_{\gamma \gamma}=a_{\sigma(\gamma) \sigma(\gamma)}$ для всех $\gamma \in \bar{\gamma}$;

(b) $a_{i \alpha}=0$ для всех $\alpha \in \bar{\alpha}, i \notin \bar{\alpha}$;

(c) $a_{j \beta}=0$ для всех $\beta \in \bar{\beta}$ u $j \notin \bar{\beta} \cup \bar{\gamma} \cup \sigma(\bar{\gamma})$;

(d) $a_{\gamma \beta}=a_{\sigma(\gamma) \beta}$ для всех $\beta \in \bar{\beta} u \gamma \in \bar{\gamma}, \gamma<\beta$;

(e) $a_{j \gamma}=-a_{j \sigma(\gamma)}$ для всех $j \notin \bar{\beta} \cup \bar{\gamma} \cup \sigma(\bar{\gamma}) u \gamma \in \bar{\gamma}$;

(f) для всех $\gamma_{1}, \gamma_{2} \in \bar{\gamma}, \gamma_{1}<\gamma_{2}$ имеет место один из следующих случаев:

- $\sigma\left(\gamma_{2}\right)<\sigma\left(\gamma_{1}\right)<\gamma_{1}<\gamma_{2}$ : тогда $a_{\gamma_{1} \gamma_{2}}=a_{\sigma\left(\gamma_{1}\right) \gamma_{2}}=a_{\sigma\left(\gamma_{2}\right) \gamma_{1}}=a_{\sigma\left(\gamma_{1}\right) \sigma\left(\gamma_{2}\right)}=0$;

- $\sigma\left(\gamma_{1}\right)<\sigma\left(\gamma_{2}\right)<\gamma_{1}<\gamma_{2}$ : moгдa $a_{\sigma\left(\gamma_{2}\right) \gamma_{1}}=a_{\sigma\left(\gamma_{1}\right) \gamma_{2}}=0, a_{\gamma_{1} \gamma_{2}}=a_{\sigma\left(\gamma_{1}\right) \sigma\left(\gamma_{2}\right)}$;

- $\sigma\left(\gamma_{1}\right)<\gamma_{1}<\sigma\left(\gamma_{2}\right)<\gamma_{2}$ : moгda $a_{\sigma\left(\gamma_{1}\right) \gamma_{2}}=0, a_{\gamma_{1} \gamma_{2}}+a_{\gamma_{1} \sigma\left(\gamma_{2}\right)}=a_{\sigma\left(\gamma_{1}\right) \sigma\left(\gamma_{2}\right)}$.

Следствие 3. Стабилизатор конфигуращии $(U, W)$ есть полупрямое произведение торической и унипотентной частей:

$$
B_{(U, W)}=T_{(U, W)} \ltimes U_{(U, W)},
$$


где $T_{(U, W)}$ есть подгруппа в группе невырожденных диагональных матрии, определяемая условиями (а), так что $\operatorname{dim} T_{(U, W)}=n-\# \bar{\gamma}$, a $U_{(U, W)}$ есть подгруппа в группе унитреугольных матрии, определяемая условиями (b)-(f).

Определение. Коразмерность торической части стабилизатора называется рангом конфигурации (или соответствующей $B$-орбиты):

$$
\operatorname{rk}(U, W):=n-\operatorname{dim} T_{(U, W)}=\# \bar{\gamma} .
$$

Доказательство предложения 2. Прежде всего, стабилизатор $B_{(U, W)}$ является подгруппой в $B$, а следовательно, состоит из верхнетреугольных матриц.

Далее, он сохраняет подпространство $U=\left\langle v_{\alpha_{1}}, \ldots, v_{\alpha_{k}}\right\rangle$. Это значит, что всякий элемент $A \in B_{(U, W)}$ переводит каждый из $v_{\alpha_{i}}$ в линейную комбинацию элементов $v_{\alpha_{j}}$, и поэтому все матричные элементы $a_{i \alpha}$, где $\alpha \in \bar{\alpha}, i \notin \bar{\alpha}$, равны нулю. (Заметим, что нули в матрице $A$ сами образуют повернутую на $90^{\circ}$ по часовой стрелке диаграмму Юнга, соответствующую подпространству $U$. В частности, это доказывает, что размерность клетки Шуберта в грассманиане равна числу клеток в соответствующей диаграмме Юнга).

Итак, клетки первой диаграммы Юнга находятся во взаимно однозначном соответствии с линейными уравнениями, задающими $B_{U}$ в группе верхнетреугольных матриц: клетка, расположенная над $i$-м (горизонтальным) звеном и слева от $j$-го (вертикального) звена (обозначим ее через $(i, j))$ соответствует уравнению $a_{i j}=0$.

Аналогично, стабилизатор нашей конфигурации сохраняет подпространство $W$. Это дает другой набор линейных уравнений на матричные элементы $a_{i j}$, и число этих уравнений равно числу клеток во второй диаграмме соответствующей отмеченной пары. Мы снова можем установить взаимно однозначное соответствие между клетками этой диаграммы и полученными уравнениями, обозначая клетки диаграммы, как в предыдущем абзаце. Вот это соответствие:

- $a_{j \beta}=0$ для всех $\beta \in \bar{\beta}$ и $j \notin \bar{\beta} \cap \bar{\gamma} \cap \sigma(\bar{\gamma}), j<\beta$; это соответствует клетке $(j, \beta)$;

- $a_{j \gamma}=-a_{j \sigma(\gamma)}$ для всех $j \notin \bar{\beta} \cup \bar{\gamma} \cup \sigma(\bar{\gamma})$ и $\gamma \in \bar{\gamma}, j<\gamma$; соответствующая клетка - это $(j, \gamma)$;

- $a_{\sigma(\gamma) \gamma}+a_{\gamma \gamma}-a_{\sigma(\gamma) \sigma(\gamma)}=0$ при всех $\gamma \in \bar{\gamma}$; соответствующая клетка это $(\sigma(\gamma), \gamma)$;

- $a_{\gamma \beta}=a_{\sigma(\gamma) \beta}$ при всех $\beta \in \bar{\beta}$ и $\gamma \in \bar{\gamma}, \gamma<\beta$; это соответствует клетке $(\sigma(\gamma), \beta)$

- $a_{\sigma\left(\gamma_{1}\right) \sigma\left(\gamma_{2}\right)}+a_{\sigma\left(\gamma_{1}\right) \gamma_{2}}=a_{\gamma_{1} \sigma\left(\gamma_{2}\right)}+a_{\gamma_{1} \gamma_{2}}$ для всех $\gamma_{1}<\gamma_{2}$; это уравнение соответствует клетке $\left(\sigma\left(\gamma_{1}\right), \gamma_{2}\right)$.

Это доказывает предложение.

Найдя стабилизатор конфигурации, мы можем отыскать его размерность, а значит, и размерность орбиты $B(U, W) \subset X$. Анализируя приведенные выше уравнения, можно найти комбинаторную интерпретацию размерности в терминах отмеченных диаграмм Юнга.

Для этого введем еще одно комбинаторное понятие. Пусть имеются два прямоугольника размера $k \times(n-k)$ и $l \times(n-l)$ соответственно и путь в каждом из них, ограничивающий диаграмму Юнга (оба пути имеют длину $n$ ). Рассмотрим множество всех чисел $i$, для которых $i$-е звенья обоих путей горизонтальны, и 
рассмотрим столбцы в диаграммах, лежащие над этими звеньями. Затем сделаем то же самое для пар «одновременно вертикальных» звеньев и рассмотрим строки слева от них.

Пересечение этих строк и столбцов также образует диаграмму Юнга. Назовем ее общей диаграммой, соответствующей данной паре диаграмм.

Пример. Паре $\left(Y_{1}, Y_{2}\right)$ диаграмм Юнга
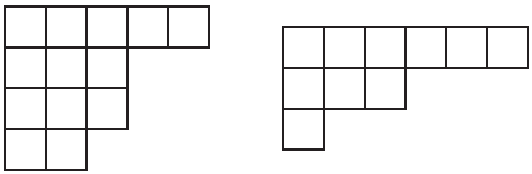

соответствует следующая общая диаграмма $Y_{\text {com }}$ :

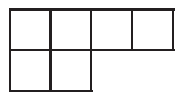

Согласно нашей конструкции отмеченных пар, точки могут содержаться только в общей диаграмме отмеченной пары.

Следствие 4. Пусть $(U, W)$ - конфигуращия подпространств, которой соответствует отмеченная пара диаграмм Юнга $\left(Y_{1}, Y_{2}\right)$ с точками в некоторых клетках диаграммъ $Y_{\text {сот }}$.

Углом с вершиной в данной клетке будем называть фигуру, образованную этой клеткой и всеми клетками, находящимися над ней в том же столб-

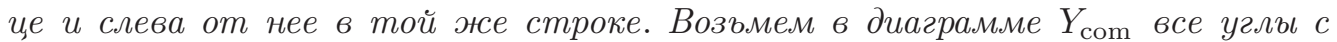
вершинами в клетках, содержащих точки. Пусть $H$ - множество клеток, принадлежсащих хотя бъ одному из этих углов. Тогда размерность В-орбитъ конфигуращии $(U, W)$ равна

$$
\operatorname{dim} B(U, W)=\# Y_{1}+\# Y_{2}-\# Y_{\mathrm{com}}+\# H,
$$

где через \#Y обозначено количество клеток в диаграмме $Y$.

Замечание. \#H равняется общему числу клеток, содержащихся во всех углах, а не сумме длин углов. Это означает, что клетку, содержащуюся в двух углах, следует считать один, а не два раза.

Доказательство следствия 4. В доказательстве предложения 2 рассматриваются две системы линейных уравнений на матричные элементы $a_{i j}$. Эти системы задают стабилизаторы подпространств $U$ и $W$ и состоят из \# $Y_{1}$ и \# $Y_{2}$ уравнений соответственно. Легко видеть, что уравнения, соответствующие клетке $(i, j)$, совпадают в обеих системах, если клетка $(i, j)$ общей диаграммы не содержится ни в каком угле, а также что система уравнений, полученная в результате объединения этих двух систем, линейно независима. Поэтому коразмерность стабилизатора $B_{(U, W)}$ в $B$ (т.е. размерность орбиты $B(U, W)$ ) равна $\# Y_{1}+\# Y_{2}-\# Y_{\text {com }}+\# H$.

Пример. Пусть общая диаграмма для отмеченной пары имеет вид

\begin{tabular}{|c|c|c|c|c|c|}
\hline$*$ & & & $*$ & & $*$ \\
\hline * & $*$ & * & $*$ & $*$ & • \\
\hline • & & & $*$ & & \\
\hline$*$ & $*$ & $*$ & • & & \\
\hline
\end{tabular}


Тогда $\# Y_{\text {сот }}=26, \# H=15$ ( $H$ состоит из всех клеток, отмеченных точкой или звездочкой).

В частности, формула для размерности позволяет описать наименьшую (т. е. наиболее вырожденную) и наибольшую орбиты. Наименьшая орбита нульмерна и соответствует паре пустых диаграмм. Она состоит из одной точки $\left(\left\langle v_{1}, \ldots, v_{k}\right\rangle\right.$, $\left.\left\langle v_{1}, \ldots, v_{l}\right\rangle\right) \in X$. Обе диаграммы Юнга, соответствующие наибольшей орбите, являются прямоугольниками размера $k \times(n-k)$ и $l \times(n-l)$ соответственно. Их общая диаграмма также есть прямоугольник размера $\min \{k, l\} \times(n-\max \{k, l\})$, точки в котором располагаются по главной диагонали, начинающейся в нижнем правом углу.

Пример. Для $n=8, k=3$ и $l=4$ наибольшей орбите соответствует такая отмеченная пара:

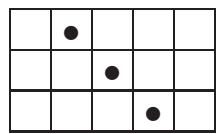

$Y_{1}$

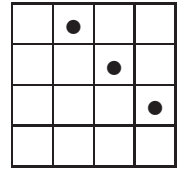

$Y_{2}$

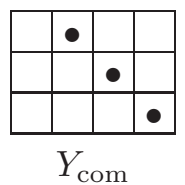

$Y_{\text {com }}$

2.4. Разложение многообразия $X$ в объединение $\mathrm{GL}(V)$-орбит. $\mathrm{GL}(V)$-орбиты в $X$ допускают существенно более простое описание: они параметризуются только одним целым положительным числом, а именно, размерностью пересечения подпространств $U$ и $W$. Для этого числа (обозначим его через $i$ ) имеются неравенства

$$
\max \{0, k+l-n\} \leqslant i \leqslant \min \{k, l\} .
$$

Обозначим соответствующую $\mathrm{GL}(V)$-орбиту через $X_{i}$ :

$$
X=\bigsqcup_{i \in[\max \{0, k+l-n\}, \min \{k, l\}]} X_{i} .
$$

Из конструкции комбинаторных данных, соответствующих $B$-орбитам, следует, что размерность пересечения $\operatorname{dim}(U \cap W)$ равна $\#(\bar{\alpha} \cap \bar{\beta})$.

\section{§3. Слабый порядок на множестве орбит}

В предыдущем параграфе мы описали множество $B$-орбит в $\operatorname{Gr}(k, V) \times \operatorname{Gr}(l, V)$. На этом множестве существует несколько структур частичного порядка. Первая, и наиболее естественная, описывается так:

Определение. Пусть $\mathcal{O}$ и $\mathcal{O}^{\prime}-$ две $B$-орбиты в $\operatorname{Gr}(k, V) \times \operatorname{Gr}(l, V)$. Скажем, что $\mathcal{O}$ не превосходит $\mathcal{O}^{\prime}$ относительно сильного (или топологического) поряд$\kappa a$, если $\mathcal{O} \subset \overline{\mathcal{O}}^{\prime}$ (здесь и далее черта над символом обозначает замыкание в топологии Зарисского). Обозначение: $\mathcal{O} \leqslant \mathcal{O}^{\prime}$.

На этом множестве существует и другой порядок, обычно называемый слабым. Обозначения, используемые ниже, взяты из работы [1].

Пусть $W$ - группа Вейля для $\mathrm{GL}(n)$, а $\Delta$ - соответствующая система корней. Обозначим простые отражения через $s_{1}, \ldots, s_{n-1}$, а соответствующие простые корни через $\alpha_{1}, \ldots, \alpha_{n-1}$. Пусть $P_{i}=B \cup B s_{i} B-$ минимальная параболическая подгруппа в $\mathrm{GL}(V)$, соответствующая простому корню $\alpha_{i}$. 
Будем говорить, что $\alpha_{i}$ nоднимает орбиту $\mathcal{O}$ до орбиты $\mathcal{O}^{\prime}$, если $\overline{\mathcal{O}}^{\prime}=$ $P_{i} \overline{\mathcal{O}} \neq \overline{\mathcal{O}}$. В этом случае $\operatorname{dim} \mathcal{O}^{\prime}=\operatorname{dim} \mathcal{O}+1$. Это понятие позволит нам определить слабый порядок.

Определение. Орбита $\mathcal{O}$ меньше или равна орбите $\mathcal{O}^{\prime}$ относительно слабого порлдка (обозначение: $\mathcal{O} \preceq \mathcal{O}^{\prime}$ ), если $\overline{\mathcal{O}}^{\prime}$ может быть получена как результат нескольких последовательных «поднятий» замыкания орбиты $\mathcal{O}$ при помощи минимальных параболических подгрупп:

$$
\mathcal{O} \preceq \mathcal{O}^{\prime} \Longleftrightarrow \exists\left(i_{1}, \ldots, i_{r}\right): \overline{\mathcal{O}}^{\prime}=P_{i_{r}} \ldots P_{i_{1}} \overline{\mathcal{O}}=\overline{P_{i_{r}} \ldots P_{i_{1}} \mathcal{O}} .
$$

Будем представлять это отношение порядка при помощи ориентированного графа. Рассмотрим граф $\Gamma(X)$, вершинами которого являются $B$-орбиты в $X$. Соединим $\mathcal{O}$ и $\mathcal{O}^{\prime}$ ребром с меткой $i$, направленным к $\mathcal{O}^{\prime}$, если $P_{i}$ поднимает $\mathcal{O}$ до орбиты $\mathcal{O}^{\prime}$.

Ясно, что каждая связная компонента графа $\Gamma(X)$ состоит из всех орбит, принадлежащих одной и той же $\mathrm{GL}(V)$-орбите $X_{i}$, и что во всякой связной компоненте имеется наибольший элемент, т. е. $B$-орбита, открытая в $X_{i}$.

Наша следующая цель состоит в описании минимальных элементов относительно слабого порядка в каждой из связных компонент.

\section{1. Комбинаторное описание действия минимальных параболиче-} ских подгрупп. Рассмотрим орбиту $\mathcal{O}$ и соответствующие ей комбинаторные данные: множества $\bar{\alpha}, \bar{\beta}, \bar{\gamma}$ и инволюцию $\sigma \in S_{n}$. Пусть минимальная параболическая подгруппа $P_{i}=B \cup B s_{i} B$ поднимает орбиту $\mathcal{O}$ до орбиты $\mathcal{O}^{\prime} \neq \mathcal{O}$. Опишем комбинаторные данные $\left(\bar{\alpha}^{\prime}, \bar{\beta}^{\prime}, \bar{\gamma}^{\prime}, \sigma^{\prime}\right)$ для орбиты $\mathcal{O}^{\prime}$.

Обозначим простую транспозицию $(i, i+1) \in S_{n}$ через $\tau_{i}$.

Возможны следующие случаи:

1. Пусть

$$
i \in \bar{\alpha}, \quad i \notin \bar{\beta}, \quad i+1 \notin \bar{\alpha}, \quad i+1 \in \bar{\beta}
$$

или, наоборот,

$$
i \notin \bar{\alpha}, \quad i \in \bar{\beta}, \quad i+1 \in \bar{\alpha}, \quad i+1 \notin \bar{\beta} .
$$

Эти два варианта соответствуют двум орбитам, которые могут быть подняты при помощи $P_{i}$ до $\mathcal{O}^{\prime}$. В этом случае комбинаторные данные для $\mathcal{O}^{\prime}$ выглядят так:

$$
\bar{\alpha}^{\prime}=\bar{\alpha} \cup\{i+1\} \backslash\{i\}, \quad \bar{\beta}^{\prime}=\bar{\beta} \backslash\{i, i+1\}, \quad \bar{\gamma}^{\prime}=\bar{\gamma} \cup\{i+1\}, \quad \sigma^{\prime}=\sigma \cdot \tau_{i} .
$$

Отметим, что $\operatorname{rk} \mathcal{O}^{\prime}=\operatorname{rk} \mathcal{O}+1, \operatorname{dim} \mathcal{O}^{\prime}=\operatorname{dim} \mathcal{O}+1$.

На языке отмеченных пар диаграмм это можно представить так. Если $i$-е и $(i+1)$-е звенья пути, который ограничивает первую диаграмму, образуют углубление (т.е. первое из них вертикально, а второе горизонтально), а соответствующие звенья для второй диаграммы образуют выступ (или же, наоборот, на данном месте в первой диаграмме имеется выступ, а во второй - углубление), то обе эти пары звеньев заменяются на выступ, ограничивающий клетку с точкой. 
Пример. Применим минимальную параболическую подгруппу $P_{2}$ к орбите $\mathcal{O} \subset \operatorname{Gr}(3,7) \times \operatorname{Gr}(4,7)$, задаваемой отмеченной парой диаграмм
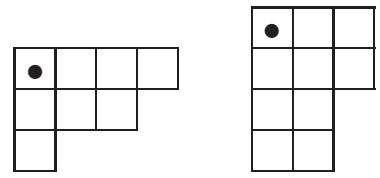

Полученная в результате поднятия орбита $\mathcal{O}^{\prime}$ будет задаваться отмеченной парой
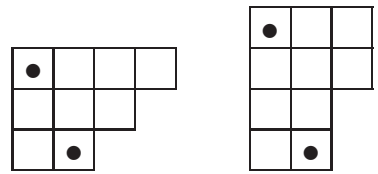

2. Во всех остальных случаях $\bar{\alpha}^{\prime}=\tau_{i}(\bar{\alpha}), \bar{\beta}^{\prime}=\tau_{i}(\bar{\beta}), \bar{\gamma}^{\prime}=\tau_{i}(\bar{\gamma})$, а перестановка $\sigma^{\prime}$ есть результат сопряжения перестановки $\sigma$ при помощи $\tau_{i}$ :

$$
\tilde{\sigma}=\tau_{i} \sigma \tau_{i} .
$$

Ранги исходной и полученной орбит равны: $\operatorname{rk} \mathcal{O}^{\prime}=\operatorname{rk} \mathcal{O}$.

\section{2. Минимальные орбиты.}

Лемма 5. Все минимальные относительно слабого порядка В-орбиты в данной $\mathrm{GL}(V)$-орбите имеют ранг 0.

Доказательство. Предположим противное. Пусть $\mathcal{O}$ - минимальная орбита положительного ранга, и пусть ей соответствует набор комбинаторных данных $(\bar{\alpha}, \bar{\beta}, \bar{\gamma}, \sigma)$, причем $\sigma \neq \mathrm{Id}$. Пусть $p \in \bar{\gamma}, p^{\prime}=\sigma(p)$. Без потери общности предположим, что ни для какого другого $q \in \bar{\gamma}$ не выполнено условие $p<q<\sigma\left(q^{\prime}\right)<p^{\prime}$.

Обозначим через $C_{1}$ множество выступов в первой диаграмме, расположенных между $p$-м и $p^{\prime}$-м звеньями, т. е. множество индексов $i, p \leqslant i<p^{\prime}$, для которых $i$-е звено в первой диаграмме горизонтально, а $(i+1)$-е вертикально. Пусть также $D_{1}$ есть множество углублений т. е. таких $i$, что $p \leqslant i<p^{\prime}$ и при этом $i$-е звено вертикально, а $(i+1)$-е горизонтально.

Обозначим аналогичные множества для второй диаграммы через $C_{2}$ и $D_{2}$. Заметим, что $\# C_{1}=\# D_{1}+1$ и $\# C_{2}=\# D_{2}+1$, поскольку в обеих диаграммах $p$-е звенья горизонтальны, а $p^{\prime}$-е вертикальны.

Теперь рассмотрим такое число $j$, что $j \in\left(C_{1} \backslash D_{2}\right) \cup\left(C_{2} \backslash D_{1}\right)$. При помощи рассуждений из разд. 3.1 можно показать, что $\overline{\mathcal{O}}=P_{j} \overline{\mathcal{O}^{\prime}}$ для некоторой орбиты $\mathcal{O}^{\prime}$. Действительно, предъявим эту орбиту.

Если перестановка $\sigma$ содержит транспозицию $(j, j+1)$, то комбинаторными данными для орбиты $\mathcal{O}^{\prime}$ будут

$$
\bar{\alpha}^{\prime}=\bar{\alpha} \cup\{j\} \backslash\{j+1\}, \quad \bar{\beta}^{\prime}=\bar{\beta} \cup\{j\}, \quad \bar{\gamma}^{\prime}=\bar{\gamma} \backslash\{j+1\}, \quad \sigma^{\prime}=\sigma \cdot \tau_{j} .
$$

В противном случае $\bar{\alpha}^{\prime}=\tau_{j}(\bar{\alpha}), \bar{\beta}^{\prime}=\tau_{j}(\bar{\beta}), \bar{\gamma}^{\prime}=\tau_{j}(\bar{\gamma}), \sigma^{\prime}=\tau_{j} \sigma \tau_{j}$.

Подсчет размерностей показывает, что $\operatorname{dim} \mathcal{O}^{\prime}=\operatorname{dim} \mathcal{O}-1$.

Остается показать, что множество $\left(C_{1} \backslash D_{2}\right) \cup\left(C_{2} \backslash D_{1}\right)$ непусто:

$$
\begin{aligned}
\#\left(\left(C_{1} \backslash D_{2}\right) \cup\left(C_{2} \backslash D_{1}\right)\right) & \geqslant \max \left(\#\left(C_{1} \backslash D_{2}\right), \#\left(C_{2} \backslash D_{1}\right)\right) \\
& \geqslant \max \left(\# C_{1}-\# C_{2}+1, \# C_{2}-\# C_{1}+1\right) \geqslant 1 .
\end{aligned}
$$


После этого можно найти все минимальные орбиты в $X_{d}$. Легко видеть, что им соответствуют следующие комбинаторные данные:

$$
\bar{\alpha} \cup \bar{\beta}=\{1, \ldots, k+l-d\}, \quad \bar{\alpha} \cap \bar{\beta}=\{1, \ldots, d\}, \quad \bar{\gamma}=\varnothing, \quad \sigma=\mathrm{Id} .
$$

Размерности всех минимальных орбит в $X_{d}$ равны $(k-d)(l-d)$. В частности, это означает, что они все замкнуты в $X_{d}$ (поскольку $X_{d}$ не содержит орбит меньших размерностей). Они соответствуют разложениям множества $\{d+1, \ldots, k+l-d\}$ на два непересекающихся подмножества, $\bar{\alpha} \backslash \bar{\beta}$ и $\bar{\beta} \backslash \bar{\alpha}$. Поэтому их число равно биномиальному коэффициенту $\left(\begin{array}{c}k+l-2 d \\ k-d\end{array}\right)$.

Также заметим, что пара диаграмм Юнга, соответствующая минимальной орбите, является дополнительной: эти две диаграммы, будучи сложенными вместе, образуют прямоугольник размера $(k-d) \times(l-d)$.

Поскольку общая диаграмма для каждой из пар диаграмм, соответствующих минимальным орбитам, пуста, то все минимальные $B$-орбиты также являются $(B \times B)$-орбитами, т. е. прямыми произведениями клеток Шуберта, взятых в каждом из двух грассманианов.

Эти результаты могут быть подытожены в виде следующей теоремы.

Теорема 6. В каждом $X_{d}$, где $d \in[\max \{k+l-n, 0\}, \min \{k, l\}]$, содержнтся $\left(\begin{array}{c}k+l-2 d \\ k-d\end{array}\right)$ минимальных орбит. Они все замкнуть в $X_{d}$, имеют размерность $(k-d)(l-d)$ и являются прямыми произведениями клеток Шуберта.

\section{§4. Разрешения особенностей для замыканий орбит}

В этом параграфе строятся разрешения особенностей для замыканий $B$-орбит в $X$.

Для минимальной параболической подгруппы $P_{i}$ и замыкания орбиты $\overline{\mathcal{O}}$ рассмотрим морфизм

$$
F_{i}: P_{i} \times{ }^{B} \overline{\mathcal{O}} \rightarrow P_{i} \overline{\mathcal{O}}, \quad(p, x) \mapsto p x .
$$

Предположим, что $\overline{\mathcal{O}} \neq P_{i} \overline{\mathcal{O}}$. В работах Кнопа [5] и Ричардсона-Спрингера [9] показано, что при этом имеет место один из трех случаев:

- Тип U: $P_{i} \mathcal{O}=\mathcal{O}^{\prime} \sqcup \mathcal{O}$ и $F_{i}$ бирационален;

- Тип N: $P_{i} \mathcal{O}=\mathcal{O}^{\prime} \sqcup \mathcal{O}$ и $F_{i}$ имеет степень 2;

- Тип Т: $P_{i} \mathcal{O}=\mathcal{O}^{\prime} \sqcup \mathcal{O} \sqcup \mathcal{O}^{\prime \prime}$ и $F_{i}$ бирационален. В этом случае $\operatorname{dim} \mathcal{O}^{\prime \prime}=$ $\operatorname{dim} \mathcal{O}$.

Оказывается, что в нашей ситуации тип $\mathrm{N}$ невозможен.

Предложение 7. Пусть $\mathcal{O}$ есть $B$-орбита в $X$ и $P_{i}$ - такая минимальная параболическая подгруппа, что $P_{i} \mathcal{O} \neq \mathcal{O}$. Тогда отображение $F_{i}: P_{i} \times{ }^{B} \mathcal{O} \rightarrow P_{i} \mathcal{O}$ бирационально.

Доказательство. Выберем канонический представитель $x$ орбиты $\mathcal{O}$, как это было сделано в предложении 1. Непосредственное вычисление показывает, что стабилизатор элемента $x$ в $P_{i}$ равняется его стабилизатору в $B$, описанному в предложении 2. Отсюда следует бирациональность отображения $F_{i}$.

Замечание. Два оставшихся типа соответствуют двум случаям, описанным в разд. 3.1: Т соответствует п. 1, а U - п. 2. В первом случае ранг орбиты увеличивается на 1, во втором остается неизменным. Поэтому слабый порядок совместим с функцией ранга: если $\mathcal{O} \preceq \mathcal{O}^{\prime}$, то rk $\mathcal{O} \leqslant \mathrm{rk} \mathcal{O}^{\prime}$. Это верно и для про- 
извольных сферических многообразий, см., например, [1]. Отметим, что сильный порядок с функцией ранга не совместим.

Предложение 7 вместе с теоремой 6 позволяет построить разрешения особенностей для $\overline{\mathcal{O}}$, аналогичные разрешениям Ботта-Самельсона для многообразий Шуберта в грассманианах.

Пусть дана орбита $\mathcal{O}$. Рассмотрим произвольную минимальную орбиту $\mathcal{O}_{\min }$, меньшую либо равную $\mathcal{O}$ в смысле слабого порядка. Это значит, что существует последовательность минимальных параболических подгрупп $\left(P_{i_{1}}, \ldots, P_{i_{r}}\right)$, для которой

$$
\overline{\mathcal{O}}=P_{i_{r}} \cdots P_{i_{1}} \overline{\mathcal{O}}_{\min } .
$$

Рассмотрим отображение

$$
F: P_{i_{r}} \times{ }^{B} \cdots \times{ }^{B} P_{i_{1}} \times{ }^{B} \overline{\mathcal{O}}_{\text {min }} \rightarrow \overline{\mathcal{O}}, \quad F:\left(p_{i_{r}}, \ldots, p_{i_{1}}, x\right) \mapsto p_{i_{r}} \cdots p_{i_{1}} x .
$$

Согласно предложению 7, оно бирационально. Но это пока еще не разрешение особенностей, поскольку $\overline{\mathcal{O}}_{\min }$ может быть особым многообразием.

Второй шаг заключается в построении $B$-эквивариантного разрешения особенностей многообразия $\overline{\mathcal{O}}_{\min }$. Теорема 6 утверждает, что $\overline{\mathcal{O}}_{\min }$ может быть представлено в виде прямого произведения

$$
\overline{\mathcal{O}}_{\min }=X_{w} \times X_{v}
$$

для некоторых многообразий Шуберта $X_{w} \subset \operatorname{Gr}(k, V)$ и $X_{v} \subset \operatorname{Gr}(l, V)$.

Для $X_{w}$ и $X_{v}$ можно взять разрешение Ботта-Самельсона:

$$
F_{w}: Z_{w} \rightarrow X_{w} \quad \text { и } \quad F_{v}: Z_{v} \rightarrow X_{v} .
$$

(Подробности этого описаны, например, в [2]). Итак, мы получаем разрешение

$$
F_{w} \times F_{v}: Z_{w} \times Z_{w} \rightarrow X_{w} \times X_{v}=\overline{\mathcal{O}}_{\min } .
$$

Рассмотрев композицию этого отображения с отображением $F$, получаем основной результат работы:

Теорема 8. Отображсение

$$
\widetilde{F}=F \circ\left(F_{w} \times F_{v}\right): P_{i_{r}} \times{ }^{B} \cdots \times{ }^{B} P_{i_{1}} \times{ }^{B}\left(Z_{w} \times Z_{v}\right) \rightarrow \overline{\mathcal{O}}
$$

есть разрешение особенностей для многообразия $\overline{\mathcal{O}}$.

Доказательство. Уже было показано, что оба отображения $F$ и $F_{w} \times F_{v}$ суть бирациональные морфизмы. Поскольку все рассматриваемые многообразия проективны, эти морфизмы являются собственными. Многообразие $P_{i_{r}} \times{ }^{B}$ $\cdots \times{ }^{B} P_{i_{1}} \times{ }^{B}\left(Z_{w} \times Z_{v}\right)$ есть последовательность однородных $B$-расслоений с неособым слоем, и поэтому оно само является неособым.

\section{ЛитеРАТУРА}

[1] M. Brion, On orbit closures of spherical subgroups in flag varieties, Comment. Math. Helv., 76:2 (2001), 263-299.

[2] M. Brion, Lectures on the geometry of flag varieties, in: Topics in Cohomological Studies of Algebraic Varieties, Trends Math., Birkhäuser, Basel, 2005, 33-85.

[3] G. Bobiński, G. Zwara, Schubert varieties and representations of Dynkin quivers, Colloq. Math., 94:2 (2002), 285-309.

[4] У. Фултон, Таблицы Юнга и их приложения $\kappa$ теории представлений и геометрии, МЦНМО, М., 2006. 
[5] F. Knop, On the set of orbits for a Borel subgroup, Comment. Math. Helv., 70:2 (1995), 285-309.

[6] L. Manivel, Fonctions symétriques, polynômes de Schubert et lieux de dégénérescence, Cours Spécialisés, 3, Soc. Math. France, Paris, 1998.

[7] P. Magyar, J. Weyman, A. Zelevinsky, Multiple flag varieties of finite type, Adv. Math., 141:1 (1999), 97-118.

[8] S. Pin, Adhérences d'orbites des sous-groupes de Borel dans les éspaces symétriques, Thèse de doctorat, Institut Fourier, Grenoble, 2001; http://www-fourier.ujf -grenoble.fr/THESE/ps/t107.ps.

[9] R. W. Richardson, T. A. Springer, The Bruhat order on symmetric varieties, Geom. Dedicata, 35:1-3 (1990), 389-436.

Независимый московский университет

Institut Fourier

Поступило в редакцию

e-mail: smirnoff@mccme.ru

1 сентября 2006 г. 